\title{
On the convex-exponent product of logharmonic mappings
}

Zayid AbdulHadi ${ }^{1}$, Najla M Alareefi ${ }^{2}$ and Rosihan M Ali ${ }^{3 *}$

${ }^{\text {*Correspondence: }}$

rosihan@cs.usm.my

${ }^{3}$ School of Mathematical Sciences,

Universiti Sains Malaysia (USM),

Penang, 11800, Malaysia

Full list of author information is

available at the end of the article

\begin{abstract}
Sufficient conditions are obtained on two given logharmonic mappings $f_{1}$ and $f_{2}$ that ensure the product $F(z)=f_{1}^{\lambda}(z) f_{2}^{1-\lambda}(z), 0 \leq \lambda \leq 1$, is a univalent starlike logharmonic mapping. Several illustrative examples are constructed from this product.

MSC: Primary 30C35; 30C45; secondary 35Q30
\end{abstract}

Keywords: logharmonic mappings; starlike mappings; spirallike mappings

\section{Introduction}

Let $U=\{z:|z|<1\}$ be the unit disk in the complex plane $\mathbb{C}$, and let $B$ denote the set of bounded analytic functions $a$ satisfying $|a(z)|<1$ in $U$. Also let $B_{0}$ denote its subclass consisting of $a \in B$ with $a(0)=0$. A logharmonic mapping $f$ defined in $U$ is a solution of the nonlinear elliptic partial differential equation

$$
\overline{\bar{f}} \overline{\bar{f}}=a \frac{f_{z}}{f}
$$

where the second dilatation function $a$ lies in $B$. Thus the Jacobian

$$
J_{f}=\left|f_{z}\right|^{2}\left(1-|a|^{2}\right)
$$

is positive, and all non-constant logharmonic mappings are therefore sense-preserving and open in $U$. If $f$ is a non-constant logharmonic mapping that vanishes only at $z=0$, then $f$ admits the representation

$$
f(z)=z^{m}|z|^{2 \beta m} h(z) \overline{g(z)}
$$

where $m$ is a nonnegative integer, $\operatorname{Re} \beta>-1 / 2$, while $h$ and $g$ are analytic functions in $U$ satisfying $g(0)=1$ and $h(0) \neq 0$ (see [1]). The exponent $\beta$ in (1) depends only on $a(0)$ and is given by

$$
\beta=\overline{a(0)} \frac{1+a(0)}{1-|a(0)|^{2}} .
$$

@2014 AbdulHadi et al:; licensee Springer. This is an Open Access article distributed under the terms of the Creative Commons Attribution License (http://creativecommons.org/licenses/by/2.0), which permits unrestricted use, distribution, and reproduction in any medium, provided the original work is properly cited. 
Note that $f(0) \neq 0$ if and only if $m=0$, and that a univalent logharmonic mapping in $U$ vanishes at the origin if and only if $m=1$, that is, $f$ has the form

$$
f(z)=z|z|^{2 \beta} h(z) \overline{g(z)}
$$

where $\operatorname{Re} \beta>-1 / 2$ and $0 \notin(h g)(U)$. This class has been studied extensively in recent years, for instance, in [1-8], and [9].

In this case, $F(\zeta)=\log f\left(e^{\zeta}\right)$ is a univalent harmonic mapping of the half-plane $\{\zeta$ : $\operatorname{Re}(\zeta)<0\}$. Studies on univalent harmonic mappings can be found in [10-16], and [17]. Such mappings are closely related to the theory of minimal surfaces $[18,19]$.

In this work, emphasis is given on univalent and sense-preserving logharmonic mappings in $U$ with respect to $a \in B_{0}$. These mappings are of the form

$$
f(z)=z h(z) \overline{g(z)}
$$

and the class consisting of such mappings is denoted by $S_{L h}$. Also let $S_{L h}^{*}$ denote its subclass of univalent starlike logharmonic mappings. The classical family $S^{*}$ of univalent analytic starlike functions is evidently a subclass of $S_{L h}^{*}$. The representation in (2) is essential to the present work as it allows the treatment of logharmonic mappings $f$ through their associated analytic representations $h$ and $g$ (see [3-5], and [6]). For example, Abdulhadi and Abu Muhanna [5] established a connection between starlike logharmonic mappings of order $\alpha$ and starlike analytic functions of order $\alpha$.

It follows from (2) that the functions $h, g$ and the dilatation $a$ satisfy

$$
\frac{z g^{\prime}(z)}{g(z)}=a(z)\left(1+\frac{z h^{\prime}(z)}{h(z)}\right)
$$

Given an analytic function $\varphi$ with a specified geometric property and $a \in B_{0}$, a common method to construct a logharmonic mapping $f(z)=z h(z) \overline{g(z)}$ is to solve for $h$ and $g$ via the equations

$$
\frac{z h(z)}{g(z)}=\varphi(z) \quad \text { and } \quad \frac{\frac{z g^{\prime}(z)}{g(z)}}{1+\frac{z h^{\prime}(z)}{h(z)}}=a(z)
$$

Thus the solution is $f(z)=z h(z) \overline{g(z)}$ with

$$
g(z)=\exp \int_{0}^{z} \frac{a(s)}{1-a(s)} \frac{\varphi^{\prime}(s)}{\varphi(s)} d s \quad \text { and } \quad h(z)=\frac{\varphi(z) g(z)}{z}
$$

In this paper, a new logharmonic mapping with a specified property is constructed by taking product combination of two functions possessing the given property. Specifically, if $f_{1}(z)=z h_{1}(z) \overline{g_{1}(z)}$ with respect to $a_{1} \in B_{0}$, and $f_{2}(z)=z h_{2}(z) \overline{g_{2}(z)}$ with respect to $a_{2} \in B_{0}$, we construct a new univalent logharmonic mapping $F(z)=f_{1}^{\lambda}(z) f_{2}^{1-\lambda}(z), 0 \leq \lambda \leq 1$, with respect to $\mu \in B_{0}$. Sufficient conditions are obtained on $f_{1}$ and $f_{2}$ for the product combination $F(z)=f_{1}^{\lambda}(z) f_{2}^{1-\lambda}(z)$ to be starlike. We close the work by giving several examples of univalent starlike logharmonic mappings constructed from this product. 


\section{Product of logharmonic mappings}

Let $\Omega$ be a simply connected domain in $\mathbb{C}$ containing the origin. Then $\Omega$ is said to be $\alpha$-spirallike, $-\pi / 2<\alpha<\pi / 2$, if $w \exp \left(-t e^{i \alpha}\right) \in \Omega$ for all $t \geq 0$ whenever $w \in \Omega$. Evidently, $\Omega$ is starlike (with respect to the origin) if $\alpha=0$.

The following result from [6] will be needed in the sequel.

Lemma 1 Let $f(z)=z h(z) \overline{g(z)}$ be logharmonic in $U$ with $0 \notin h g(U)$. Then $f \in S_{L h}^{*}$ if and only if $\varphi(z)=z h(z) / g(z) \in S^{*}$.

Theorem 1 Let $f(z)=z h(z) \overline{g(z)} \in S_{L h}^{*}$ with respect to $a \in B_{0}$, and let $\gamma$ be a constant with $\operatorname{Re} \gamma>-1 / 2$. Then $F(z)=f(z)|f(z)|^{2 \gamma}$ is an $\alpha$-spirallike logharmonic mapping with respect to

$$
\hat{a}(z)=\frac{1+\bar{\gamma}}{1+\gamma} \frac{a(z)+\frac{\bar{\gamma}}{1+\bar{\gamma}}}{1+a(z) \frac{\gamma}{1+\gamma}}=\frac{(1+\bar{\gamma}) a(z)+\bar{\gamma}}{1+\gamma+\gamma a(z)},
$$

where $\alpha=\tan ^{-1}(2 \operatorname{Im} \gamma /(1+2 \operatorname{Re} \gamma))$.

Proof The function $F=f|f|^{2 \gamma}=f^{1+\gamma} \bar{f}^{\gamma}$ is logharmonic with respect to $\hat{a}=\left(\overline{F_{\bar{z}}} / \bar{F}\right) /\left(F_{z} / F\right)$. Indeed,

$$
\begin{aligned}
& \hat{a}(z)=\frac{(1+\bar{\gamma}) a(z) \frac{f_{\bar{z}}}{f}+\bar{\gamma}\left(\frac{\overline{\bar{f}_{\bar{z}}}}{\bar{f}}\right)}{(1+\gamma) \frac{f_{z}}{f}+\gamma \frac{\overline{\bar{f}_{\bar{z}}}}{\bar{f}}} \\
& =\frac{(1+\bar{\gamma}) a(z) \frac{f_{z}}{f}+\bar{\gamma} \frac{f_{z}}{f}}{(1+\gamma) \frac{f_{z}}{f}+\gamma a(z) \frac{f_{z}}{f}}=\frac{1+\bar{\gamma}}{1+\gamma} \frac{a(z)+\frac{\bar{\gamma}}{1+\bar{\gamma}}}{1+a(z) \frac{\gamma}{1+\gamma}} .
\end{aligned}
$$

Thus

$$
|\hat{a}(z)|=\left|\frac{a(z)+\frac{\bar{\gamma}}{1+\bar{\gamma}}}{1+a(z) \frac{\gamma}{1+\gamma}}\right|<1
$$

provided $|\gamma|^{2}<|1+\gamma|^{2}$, which evidently holds since $\operatorname{Re} \gamma>-1 / 2$.

Also $F=f|f|^{2 \gamma}=f^{1+\gamma} \bar{f}^{\gamma}=z|z|^{2 \gamma} h^{1+\gamma} g^{\gamma} \overline{h^{\bar{\gamma}}} g^{1+\bar{\gamma}}$. Let $H=h^{1+\gamma} g^{\gamma}, G=h^{\bar{\gamma}} g^{1+\bar{\gamma}}$, and $\psi(z)=$ $z H(z) / G(z)^{e^{2 i \alpha}}$. Then

$$
e^{-i \alpha} \frac{z \psi^{\prime}(z)}{\psi(z)}=e^{-i \alpha}+\left((1+\gamma) e^{-i \alpha}-\bar{\gamma} e^{i \alpha}\right) \frac{z h^{\prime}(z)}{h(z)}-\left((1+\bar{\gamma}) e^{i \alpha}-\gamma e^{-i \alpha}\right) \frac{z g^{\prime}(z)}{g(z)} .
$$

The condition on $\alpha$ ensures that

$$
\frac{(1+\gamma) e^{-i \alpha}-\bar{\gamma} e^{i \alpha}}{\cos \alpha}=\frac{(1+\bar{\gamma}) e^{i \alpha}-\gamma e^{-i \alpha}}{\cos \alpha}=1 .
$$

Also Lemma 1 shows that $\varphi(z)=z h(z) / g(z) \in S^{*}$. Thus

$$
\operatorname{Re}\left(e^{-i \alpha} \frac{z \psi^{\prime}(z)}{\psi(z)}\right)=(\cos \alpha) \operatorname{Re}\left(\frac{z \varphi^{\prime}(z)}{\varphi(z)}\right)>0
$$


and it follows from [6, Theorem 2.1] that $F$ is $\alpha$-spirallike logharmonic whose dilatation is $\hat{a}(z)$.

Remark 1 Observe that $F$ in Theorem 1 is starlike if and only if $\gamma>-1 / 2$.

Theorem 2 Let $f_{k}(z)=z h_{k}(z) \overline{g_{k}(z)} \in S_{L h}^{*}(k=1,2)$ with respect to the same $a \in B_{0}$. Then $F(z)=f_{1}^{\lambda}(z) f_{2}^{1-\lambda}(z), 0 \leq \lambda \leq 1$, is a univalent starlike logharmonic mapping with respect to the same a.

Proof Let $\mu=\left(\overline{F_{\bar{z}}} / \bar{F}\right) /\left(F_{z} / F\right)$. It follows from (3) that

$$
\begin{aligned}
& \mu=\frac{\lambda \frac{\overline{f_{1 \bar{z}}}}{\bar{f}_{1}}+(1-\lambda) \frac{\overline{f_{2 \bar{z}}}}{\lambda \frac{f_{1 z}}{f_{2}}}+(1-\lambda) \frac{f_{2 z}}{f_{2}}}{f_{1}}=\frac{\lambda \frac{g_{1}^{\prime}}{g_{1}}+(1-\lambda) \frac{g_{2}^{\prime}}{g_{2}}}{\lambda\left(\frac{\left.z h_{1}\right)^{\prime}}{\left(z h_{1}\right)}+(1-\lambda) \frac{\left(z h_{2}\right)^{\prime}}{\left(z h_{2}\right)}\right.} \\
& =\frac{\lambda a \frac{\left(z h_{1}\right)^{\prime}}{\left(z h_{1}\right)}+(1-\lambda) a \frac{\left(z h_{2}\right)^{\prime}}{\left(z h_{2}\right)}}{\lambda \frac{\left(z h_{1}\right)^{\prime}}{\left(z h_{1}\right)}+(1-\lambda) \frac{\left(z h_{2}\right)^{\prime}}{\left(z h_{2}\right)}}=a \text {. }
\end{aligned}
$$

Hence $|\mu(z)|<1$ in $U$, which implies that $F$ is a locally univalent logharmonic mapping.

Next $F$ is shown to have the form (2). Since $f_{1}=z h_{1} \overline{g_{1}}$, and $f_{2}=z h_{2} \overline{g_{2}}$, then

$$
\begin{aligned}
F(z) & =f_{1}^{\lambda}(z) f_{2}^{1-\lambda}(z)=\left(z h_{1}(z) \overline{g_{1}(z)}\right)^{\lambda}\left(z h_{2}(z) \overline{g_{2}(z)}\right)^{1-\lambda} \\
& =z h_{1}^{\lambda} h_{2}^{1-\lambda} \overline{g_{1}^{\lambda}(z) g_{2}^{1-\lambda}(z)}=z h(z) \overline{g(z)}
\end{aligned}
$$

with $h=h_{1}^{\lambda} h_{2}^{1-\lambda}$ and $g=g_{1}^{\lambda} g_{2}^{1-\lambda}$.

Since $f_{k}$ is starlike, that is, each $\varphi_{k}=z h_{k} / g_{k}$ satisfies the condition $\operatorname{Re} z \varphi_{k}^{\prime}(z) / \varphi_{k}(z)>0$ in $U$, direct computations show that

$$
\begin{aligned}
\frac{\partial \arg \left(F\left(r e^{i \theta}\right)\right)}{\partial \theta}= & \operatorname{Re}\left(\frac{z F_{z}}{F}-\frac{\bar{z} F_{\bar{z}}}{F}\right)=\lambda \operatorname{Re}\left(\frac{z f_{1 z}}{f_{1}}-\frac{\bar{z} f_{1 \bar{z}}}{f_{1}}\right) \\
& +(1-\lambda) \operatorname{Re}\left(\frac{z f_{2 z}}{f_{2}}-\frac{\bar{z} f_{2 \bar{z}}}{f_{2}}\right) \\
= & \lambda \operatorname{Re}\left(\frac{z \varphi_{1}^{\prime}(z)}{\varphi_{1}(z)}\right)+(1-\lambda) \operatorname{Re}\left(\frac{z \varphi_{2}^{\prime}(z)}{\varphi_{2}(z)}\right)>0 .
\end{aligned}
$$

Thus $F$ is starlike.

The following corollary is an immediate consequence of Theorem 2.

Corollary 1 Let $f_{k}(z)=z h_{k}(z) \overline{g_{k}(z)} \in S_{L h}^{*}(k=1,2, \ldots, n)$ with respect to the same $a \in B_{0}$. Then $F=f_{1}^{\lambda_{1}} f_{2}^{\lambda_{2}} \cdots f_{n}^{\lambda_{n}}$ is a univalent starlike logharmonic mapping with respect to the same $a$, where $0 \leq \lambda_{k} \leq 1$ and $\lambda_{1}+\lambda_{2}+\cdots+\lambda_{n}=1$.

Theorem $3 \operatorname{Let}_{k}(z)=z h_{k}(z) \overline{g_{k}(z)} \in S_{L h}^{*}(k=1,2)$ with respect to $a_{k} \in B_{0}$. Suppose also that

$$
\operatorname{Re}\left(1-a_{1} \overline{a_{2}}\right) \frac{\left(z h_{1}\right)^{\prime}}{\left(z h_{1}\right)} \overline{\left(\frac{\left(z h_{2}\right)^{\prime}}{\left(z h_{2}\right)}\right)} \geq 0 .
$$

Then $F(z)=f_{1}^{\lambda}(z) f_{2}^{1-\lambda}(z), 0 \leq \lambda \leq 1$, is a univalent starlike logharmonic mapping. 
Proof The argument is similar to the proof of Theorem 2. From (5), evidently $F$ has the form (2).

Let $\left(F_{z} / F\right) \mu(z)=\overline{F_{\bar{z}}} / \bar{F}$. Since $\left|a_{k}\right|<1$, it follows from (3) and (4) that

$$
\begin{aligned}
|\mu(z)| & =\left|\frac{\lambda \frac{g_{1}^{\prime}}{g_{1}}+(1-\lambda) \frac{g_{2}^{\prime}}{g_{2}}}{\lambda \frac{\left(z h_{1}\right)^{\prime}}{\left(z h_{1}\right)}+(1-\lambda) \frac{\left(z h_{2}\right)^{\prime}}{\left(z h_{2}\right)}}\right| \\
& =\left|\frac{\lambda a_{1} \frac{\left(z h_{1}\right)^{\prime}}{\left(z h_{1}\right)}+(1-\lambda) a_{2} \frac{\left(z h_{2}\right)^{\prime}}{\left(z h_{2}\right)}}{\lambda \frac{\left(z h_{1}\right)^{\prime}}{\left(z h_{1}\right)}+(1-\lambda) \frac{\left(z h_{2}\right)^{\prime}}{\left(z h_{2}\right)}}\right| .
\end{aligned}
$$

By assumption,

$$
\begin{gathered}
\left|\lambda \frac{\left(z h_{1}\right)^{\prime}}{\left(z h_{1}\right)}+(1-\lambda) \frac{\left(z h_{2}\right)^{\prime}}{\left(z h_{2}\right)}\right|^{2}-\left|\lambda a_{1} \frac{\left(z h_{1}\right)^{\prime}}{\left(z h_{1}\right)}+(1-\lambda) a_{2} \frac{\left(z h_{2}\right)^{\prime}}{\left(z h_{2}\right)}\right|^{2} \\
=\lambda^{2}\left(1-\left|a_{1}\right|^{2}\right)\left|\frac{\left(z h_{1}\right)^{\prime}}{\left(z h_{1}\right)}\right|^{2}+(1-\lambda)^{2}\left(1-\left|a_{2}\right|^{2}\right)\left|\frac{\left(z h_{2}\right)^{\prime}}{\left(z h_{2}\right)}\right|^{2} \\
+2 \lambda(1-\lambda) \operatorname{Re}\left(\left(1-a_{1} \overline{a_{2}}\right) \frac{\left(z h_{1}\right)^{\prime}}{\left(z h_{1}\right)} \overline{\left(\frac{\left(z h_{2}\right)^{\prime}}{\left(z h_{2}\right)}\right)}\right)>0
\end{gathered}
$$

whence $|\mu(z)|<1$, which implies that $F$ is locally univalent.

Now the associated analytic function for $F$ is given by $\varphi=\left(z h_{1}^{\lambda} h_{2}^{1-\lambda}\right) /\left(g_{1}^{\lambda} g_{2}^{1-\lambda}\right)$. Let $\varphi(U)=\Omega$. From Lemma $1, \varphi_{k}=z h_{k} / g_{k} \in S^{*}$, and thus

$$
\operatorname{Re}\left(\frac{z \varphi^{\prime}(z)}{\varphi(z)}\right)=\lambda \operatorname{Re}\left(\frac{z \varphi_{1}^{\prime}(z)}{\varphi_{1}(z)}\right)+(1-\lambda) \operatorname{Re}\left(\frac{z \varphi_{2}^{\prime}(z)}{\varphi_{2}(z)}\right)>0
$$

Hence $\Omega$ is a starlike domain, and we deduce that $F$ is a univalent starlike logharmonic mapping.

Theorem 4 Let $f_{k}=z h_{k} \overline{g_{k}} \in S_{L h}$ with respect to $a_{k} \in B, k=1,2$, satisfying $z h_{k} g_{k}=z$. Then $F(z)=f_{1}^{\lambda}(z) f_{2}^{1-\lambda}(z), 0 \leq \lambda \leq 1$, is a univalent starlike logharmonic mapping.

Proof Since

$$
\frac{\left(z h_{k}\right)^{\prime}}{\left(z h_{k}\right)}+\frac{g_{k}^{\prime}}{g_{k}}=\frac{1}{z}
$$

it follows from (3) that

$$
\frac{\left(z h_{k}\right)^{\prime}}{\left(z h_{k}\right)}=\frac{1}{z\left(1+a_{k}\right)}
$$

With $F(z)=f_{1}^{\lambda}(z) f_{2}^{1-\lambda}(z),(6)$ and (7) readily yield

$$
|\mu(z)|=\left|\frac{\lambda a_{1}+(1-\lambda) a_{2}+a_{1} a_{2}}{1+(1-\lambda) a_{1}+\lambda a_{2}}\right| .
$$

Evidently $|\mu(z)|<1$ is equivalent to $\psi(\lambda)=\left|1+(1-\lambda) a_{1}+\lambda a_{2}\right|^{2}-\left|\lambda a_{1}+(1-\lambda) a_{2}+a_{1} a_{2}\right|^{2}>0$. 
Now

$$
\begin{aligned}
\psi(\lambda)= & 2 \lambda\left(\left(1-\left|a_{1}\right|^{2}\right) \operatorname{Re} a_{2}-\left(1-\left|a_{2}\right|^{2}\right) \operatorname{Re} a_{1}-\left(\left|a_{1}\right|^{2}-\left|a_{2}\right|^{2}\right)\right) \\
& +\left(1-\left|a_{2}\right|^{2}\right)\left|1+a_{1}\right|^{2}
\end{aligned}
$$

is a continuous monotonic function of $\lambda$ in the interval $[0,1]$. Since

$$
\psi(0)=\left(1-\left|a_{2}\right|^{2}\right)\left|1+a_{1}\right|^{2}>0
$$

and

$$
\psi(1)=\left(1-\left|a_{1}\right|^{2}\right)\left|1+a_{2}\right|^{2}>0,
$$

we deduce that $\psi(\lambda)>0$ for all $\lambda \in[0,1]$, and thus $F$ is locally univalent.

With $\varphi_{k}=z h_{k} / g_{k}$, then

$$
\operatorname{Re}\left(\frac{z \varphi_{k}^{\prime}(z)}{\varphi_{k}(z)}\right)=\operatorname{Re}\left(\left(1-a_{k}\right) \frac{z\left(z h_{k}\right)^{\prime}}{\left(z h_{k}\right)}\right)=\operatorname{Re}\left(\frac{1-a_{k}}{1+a_{k}}\right)>0
$$

Hence $\varphi_{k}$ is starlike univalent, and from Lemma $1, f_{k}(z)=z h_{k}(z) \overline{g_{k}(z)}$ is starlike univalent logharmonic.

The associated analytic function for $F$ is given by $\varphi(z)=\left(z h_{1}^{\lambda} h_{2}^{1-\lambda}\right) /\left(g_{1}^{\lambda} g_{2}^{1-\lambda}\right)$. Further

$$
\operatorname{Re}\left(\frac{z \varphi^{\prime}(z)}{\varphi(z)}\right)=\lambda \operatorname{Re}\left(\frac{z \varphi_{1}^{\prime}(z)}{\varphi_{1}(z)}\right)+(1-\lambda) \operatorname{Re}\left(\frac{z \varphi_{2}^{\prime}(z)}{\varphi_{2}(z)}\right)>0,
$$

and thus $F$ is starlike.

The proof of Theorem 4 evidently gives the following result of [6, Lemma 3.1 and Theorem 3.2].

Corollary 2 Let $f_{k}=z h_{k} \overline{g_{k}} \in S_{L h}(k=1,2)$ with respect to $a_{k} \in B_{0}$, and suppose that $z h_{k} g_{k}=z$. Then $\varphi(z)=z\left(h_{k}(z)\right)^{2} \in S^{*}$.

\section{Examples}

We give several illustrative examples in this section.

\section{Example 1 Let}

$$
f(z)=z\left(\frac{1-\bar{z}}{1-z}\right)
$$

Then $f$ is a univalent logharmonic mapping with respect to $a(z)=-z$, and it maps $U$ onto $U[6]$.

Now the function $F(z)=f(z)|f(z)|^{2 \gamma}$ is an $\alpha$-spirallike logharmonic mapping with respect to

$$
\hat{a}(z)=\frac{1+\bar{\gamma}}{1+\gamma} \frac{-z+\frac{\bar{\gamma}}{1+\bar{\gamma}}}{1-z \frac{\gamma}{1+\gamma}},
$$



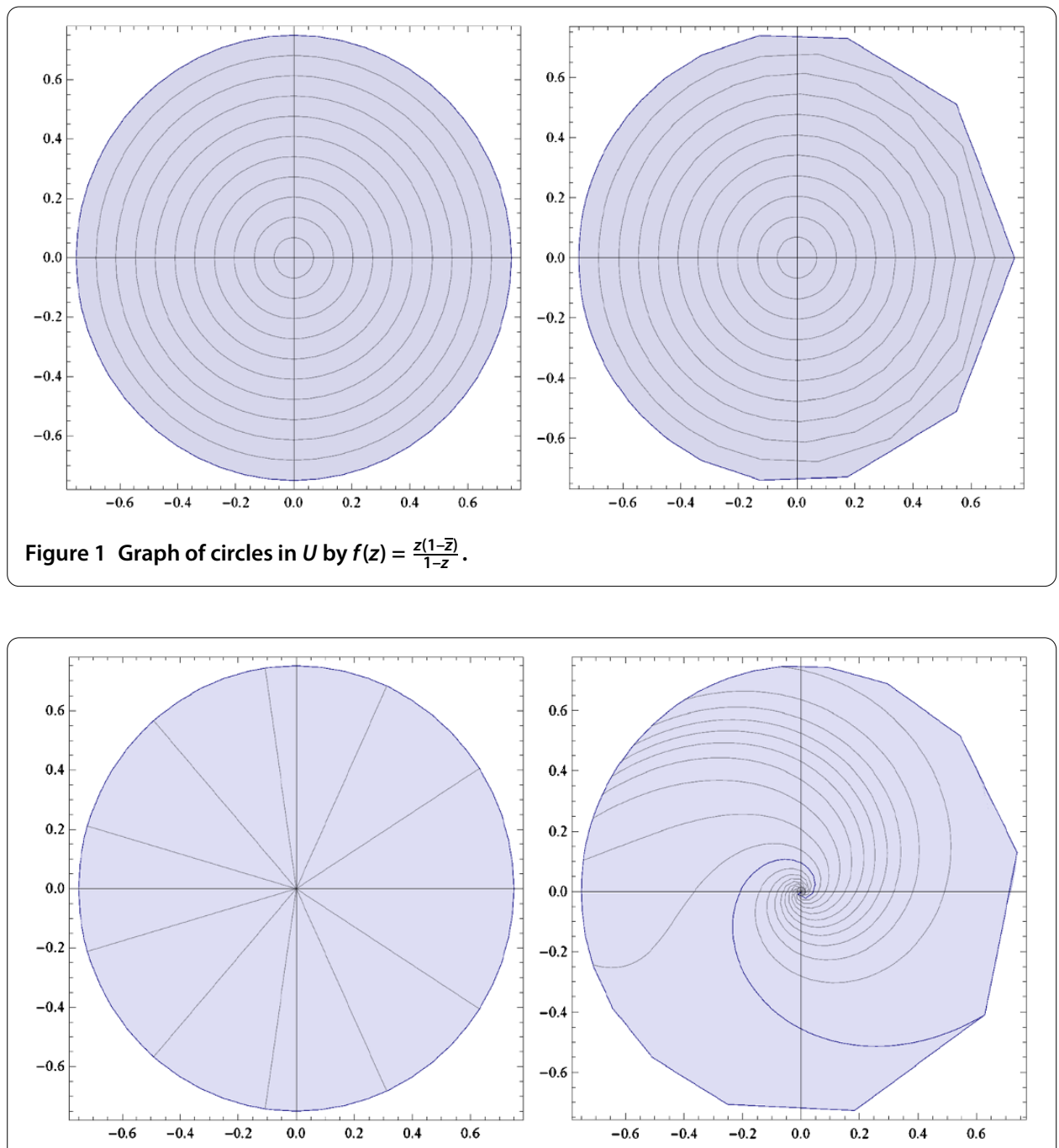

Figure 2 Graph of radial slits by $F(z)=f(z)|f(z)|^{2 i}, f(z)=\frac{z(1-\bar{z})}{1-z}$.

where $\alpha=\tan ^{-1}(2 \operatorname{Im} \gamma /(1+2 \operatorname{Re} \gamma))$. In particular, if $\gamma=i$, then $\alpha=\tan ^{-1}(2)=0.352 \pi$, and

$$
\hat{a}(z)=\frac{-i-(1-i) z}{1+i-i z}
$$

The image of circles in the unit disk under $f$ is shown in Figure 1, and Figure 2 shows the image of the radial slits in $U$ by $F$.

Example 2 Consider the functions

$$
f_{1}(z)=z\left(\frac{1-\bar{z}}{1-z}\right) \exp \left\{\operatorname{Re} \frac{4 z}{1-z}\right\} \quad \text { and } \quad f_{2}(z)=z\left(\frac{1+\bar{z}}{1+z}\right) .
$$

Since $\varphi_{1}(z)=z /(1-z)^{2}$ and $\varphi_{2}(z)=z /(1+z)^{2}$ are starlike analytic functions, it follows from [5, Theorem 1] that $f_{1}$ and $f_{2}$ are starlike logharmonic mappings with respect to $a(z)=z$. Theorem 2 shows that $F(z)=f_{1}^{\lambda}(z) f_{2}^{1-\lambda}(z), 0 \leq \lambda \leq 1$, is a starlike univalent logharmonic mapping. 


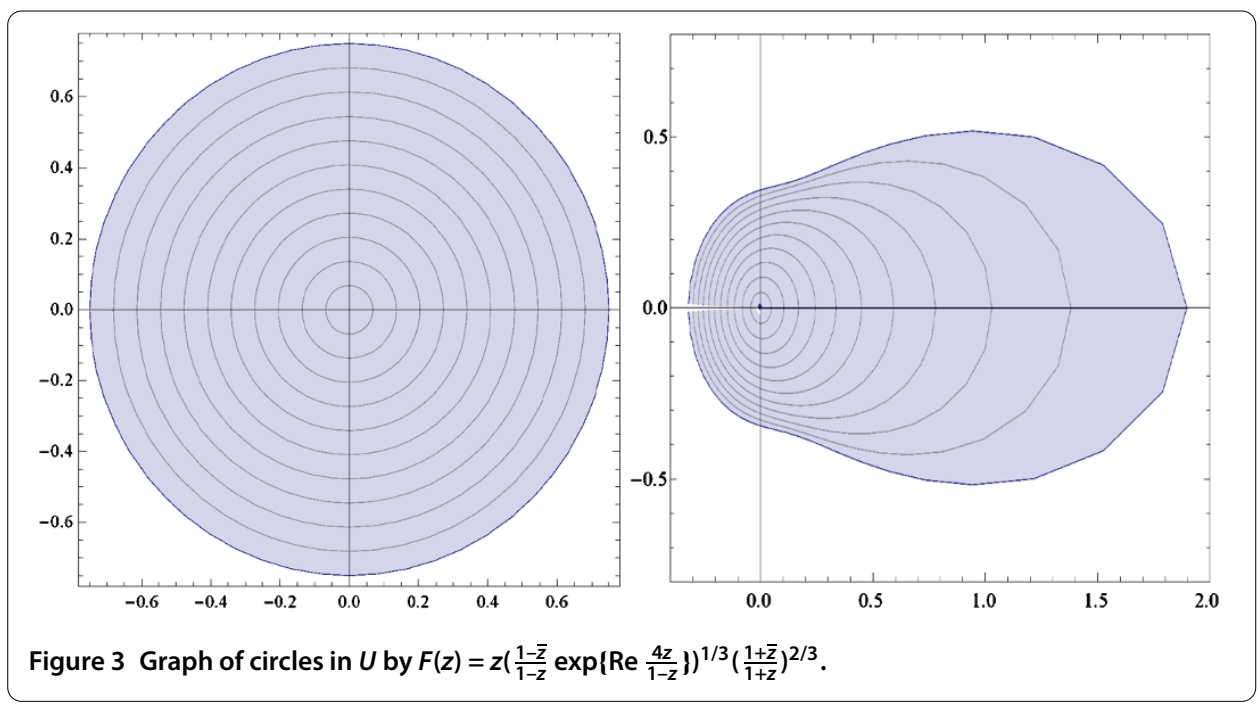

The image of $F$ is shown in Figure 3 for $\lambda=1 / 3$.

Example 3 In this example, let

$$
f_{1}(z)=z \frac{(1-\bar{z})}{(1-z)} \quad \text { and } \quad f_{2}(z)=z \frac{(1-\bar{z})}{(1-z)} \exp \left\{\operatorname{Re} \frac{4 z}{1-z}\right\}
$$

and

$$
F(z)=f_{1}^{\lambda}(z) f_{2}^{1-\lambda}(z), \quad 0 \leq \lambda \leq 1
$$

Simple calculations show that $f_{1}$ and $f_{2}$ are respectively starlike logharmonic with dilatations $a_{1}(z)=-z$ and $a_{2}(z)=z$. Also $F$ is logharmonic with respect to $\mu(z)=z((1-2 \lambda)+$ $z) /(1+(1-2 \lambda) z)$.

Since

$$
\begin{aligned}
\operatorname{Re}\left(\left(1-a_{1} \overline{a_{2}}\right) \frac{\left(z h_{1}\right)^{\prime}}{\left(z h_{1}\right)} \overline{\left.\left(\frac{\left(z h_{2}\right)^{\prime}}{\left(z h_{2}\right)}\right)\right)}\right) & =\operatorname{Re}\left(\left(1+|z|^{2}\right) \frac{1}{z(1-z)} \frac{1+\bar{z}}{\bar{z}(1-\bar{z})^{2}}\right) \\
& =\frac{\left(1+|z|^{2}\right)}{|z|^{2}|1-z|^{2}} \operatorname{Re} \frac{1+z}{1-z}>0,
\end{aligned}
$$

the conditions of Theorem 3 are satisfied, and thus $F$ is starlike univalent.

The image of circles in $U$ under $F$ for $\lambda=1 / 3$ is shown in Figure 4.

Example 4 Let $f_{1}(z)=z h_{1}(z) \overline{g_{1}(z)}$, where $z h_{1}(z) g_{1}(z)=z, a_{1}(z)=z$, and

$$
h_{1}(z)=\frac{1}{1+z}, \quad g_{1}(z)=1+z
$$

Thus

$$
f_{1}(z)=\frac{z(1+\bar{z})}{(1+z)}
$$



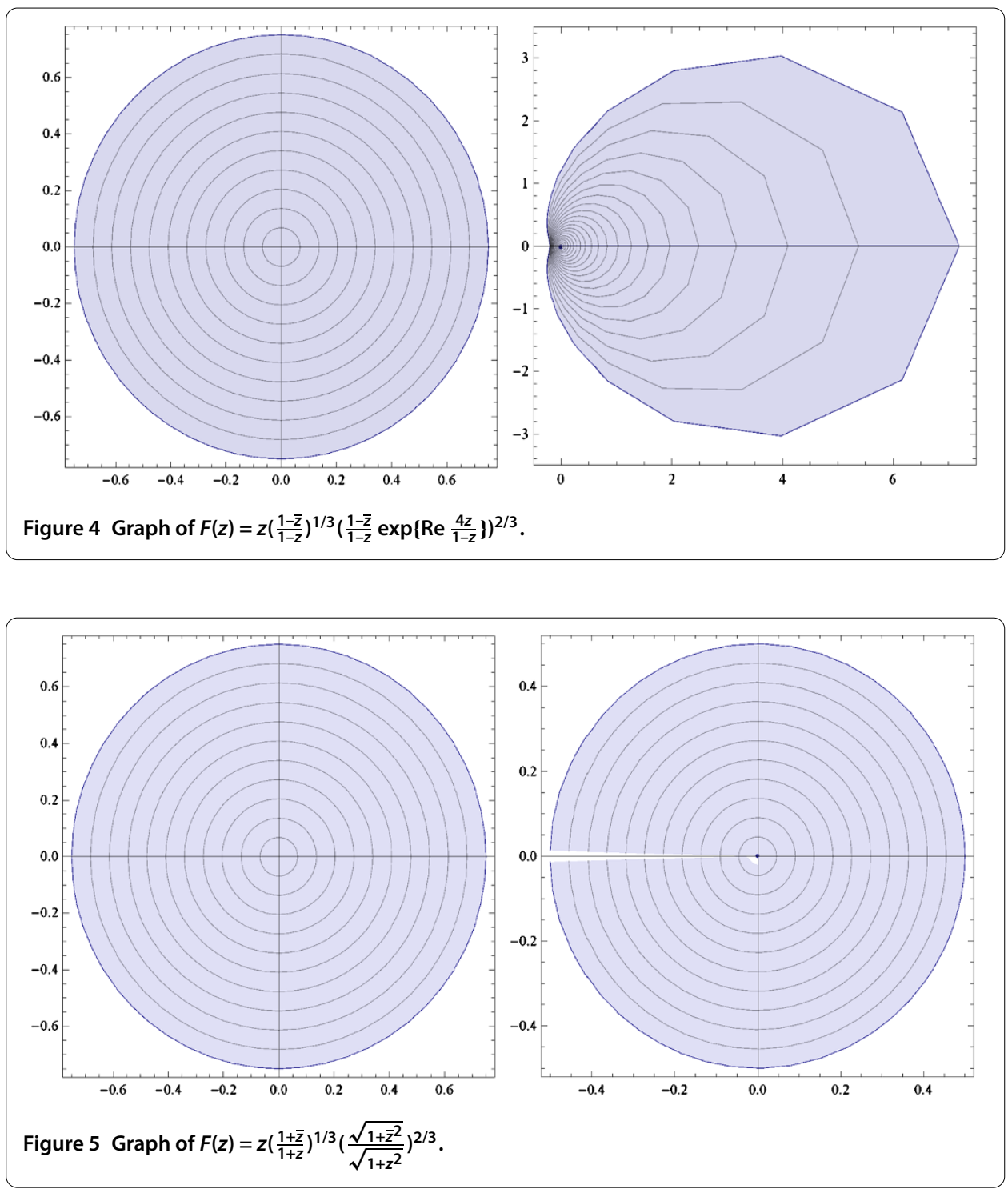

Further, let $f_{2}(z)=z h_{2}(z) \overline{g_{2}(z)}$, where $z h_{2}(z) g_{2}(z)=z, a_{2}(z)=z^{2}$, and

$$
h_{2}(z)=\frac{1}{\sqrt{1+z^{2}}}, \quad g_{2}(z)=\sqrt{1+z^{2}} .
$$

In this case,

$$
f_{2}(z)=\frac{z \sqrt{1+\bar{z}^{2}}}{\sqrt{1+z^{2}}}
$$

Since $f_{1}$ and $f_{2}$ satisfy the conditions of Theorem 4 , we deduce that $F(z)=f_{1}^{\lambda}(z) f_{2}^{1-\lambda}(z)$, $0 \leq \lambda \leq 1$, is a univalent starlike logharmonic mapping. The image of $U$ under $F$ for $\lambda=1 / 3$ is shown in Figure 5. 


\section{Competing interests}

The authors declare that they have no competing interests.

\section{Authors' contributions}

The study was conceived and planned by all authors. Every author participated in the discussions of tackling the problem and the directions of the proofs of the results. All authors read and approved the final manuscript.

\section{Author details}

${ }^{1}$ Department of Mathematics, American University of Sharjah, Sharjah, 26666, UAE. ${ }^{2}$ Department of Mathematics, University of Dammam, Dammam, 31113, Kingdom of Saudi Arabia. ${ }^{3}$ School of Mathematical Sciences, Universiti Sains Malaysia (USM), Penang, 11800, Malaysia.

\section{Acknowledgements}

This work was completed when the first author was visiting Universiti Sains Malaysia (USM). The work presented here was supported in parts by the FRGS and USM-RU research grants. The authors are thankful to the referees for the suggestions that helped improve the clarity of this manuscript.

\section{Received: 18 April 2014 Accepted: 19 November 2014 Published: 08 Dec 2014}

\section{References}

1. Abdulhadi, Z, Bshouty, D: Univalent functions in $H \cdot \bar{H}(D)$. Trans. Am. Math. Soc. 305(2), 841-849 (1988)

2. Abdulhadi, Z, Ali, RM: Univalent logharmonic mappings in the plane. Abstr. Appl. Anal. 2012, Article ID 721943 (2012)

3. Abdulhadi, Z: Close-to-starlike logharmonic mappings. Int. J. Math. Math. Sci. 19(3), 563-574 (1996)

4. Abdulhadi, Z: Typically real logharmonic mappings. Int. J. Math. Math. Sci. 31(1), 1-9 (2002)

5. Abdulhadi, Z, Abu Muhanna, Y: Starlike log-harmonic mappings of order $\alpha$. JIPAM. J. Inequal. Pure Appl. Math. 7(4), 123 (2006) (electronic)

6. Abdulhadi, Z, Hengartner, W: Spirallike logharmonic mappings. Complex Var. Theory Appl. 9(2-3), 121-130 (1987)

7. Abdulhadi, Z, Hengartner, W, Szynal, J: Univalent logharmonic ring mappings. Proc. Am. Math. Soc. 119(3), 735-745 (1993)

8. Abdulhadi, Z, Hengartner, W: One pointed univalent logharmonic mappings. J. Math. Anal. Appl. 203(2), 333-351 (1996)

9. Abdulhadi, Z, Hengartner, W: Polynomials in HW. Complex Var. Theory Appl. 46(2), 89-107 (2001)

10. Abu-Muhanna, Y, Lyzzaik, A: The boundary behaviour of harmonic univalent maps. Pac. J. Math. 141(1), 1-20 (1990)

11. Clunie, J, Sheil-Small, T: Harmonic univalent functions. Ann. Acad. Sci. Fenn., Ser. A 1 Math. 9, 3-25 (1984)

12. Duren, PL: Univalent Functions. Grundlehren der Mathematischen Wissenschaften, vol. 259. Springer, New York (1983)

13. Duren, P, Schober, G: A variational method for harmonic mappings onto convex regions. Complex Var. Theory Appl. 9(2-3), 153-168 (1987)

14. Duren, P, Schober, G: Linear extremal problems for harmonic mappings of the disk. Proc. Am. Math. Soc. 106(4), 967-973 (1989)

15. Hengartner, W, Schober, G: On the boundary behavior of orientation-preserving harmonic mappings. Complex Var. Theory Appl. 5(2-4), 197-208 (1986)

16. Hengartner, W, Schober, G: Harmonic mappings with given dilatation. J. Lond. Math. Soc. (2) 33(3), $473-483$ (1986)

17. Jun, SH: Univalent harmonic mappings on $\Delta=\{z:|z|>1\}$. Proc. Am. Math. Soc. 119(1), 109-114 (1993)

18. Nitsche, JCC: Lectures on Minimal Surfaces, vol. 1. Cambridge University Press, Cambridge (1989). Translated from the German by Jerry M Feinberg

19. Osserman, R: A Survey of Minimal Surfaces, 2nd edn. Dover, New York (1986)

10.1186/1029-242X-2014-485

Cite this article as: AbdulHadi et al.: On the convex-exponent product of logharmonic mappings. Journal of Inequalities and Applications 2014, 2014:485

\section{Submit your manuscript to a SpringerOpen ${ }^{\ominus}$ journal and benefit from:}

- Convenient online submission

Rigorous peer review

- Immediate publication on acceptance

- Open access: articles freely available online

- High visibility within the field

- Retaining the copyright to your article 